\title{
Association between insurance status and mortality in individuals with albuminuria: an observational cohort study
}

\author{
Milda R. Saunders ${ }^{1 *}$, Ana Catherine Ricardo², Jinsong Chen², Marshall H. Chin ${ }^{3}$ and James P. Lash²
}

\begin{abstract}
Background: In the general population, the association between uninsurance and mortality is well established. We sought to evaluate the association of health insurance status with mortality among working-age participants with albuminuria in the Third National Health and Nutrition Examination Survey, 1988-1994 (NHANES III).

Methods: We used data from non-elderly adult participants (18-64) of NHANES III (1988-1994), a nationally representative study of the US civilian, noninstitutionalized population, who provided information on insurance and who had albuminuria, defined as a urine albumin-to-creatinine ratio [UACR] $\geq 30 \mathrm{mg} / \mathrm{g}$ and their subsequent mortality to December 31, 2006. Cox proportional hazards models were used to determine associations between insurance status and all-cause mortality and cardiovascular mortality in patients with CKD while adjusting in a stepwise fashion for sociodemographic factors, co-morbidities, and co-morbidity severity/control covariates.

Results: In our sample of individuals with albuminuria $(n=903)$, mean estimated glomerular filtration rate (eGFR) was $101.6 \mathrm{ml} / \mathrm{min} / 1.73 \mathrm{~m}^{2}$ with $4.7 \%$ with an eGFR $<60$. Approximately $15 \%$ of the sample was uninsured, $18 \%$ had public insurance and $67 \%$ had private insurance. Compared to individuals with private insurance, those with public insurance or no insurance were significantly more likely to be a racial or ethnic minority, to have income $<200 \%$ below the federal poverty level, to have less than high school education; and they were less likely to be married and to report good or excellent health, all $p<0.05$. Being uninsured or having public insurance was associated with increased all-cause mortality in the fully adjusted model (HR 2.97 and 3.65, respectively, $p<0.05$ ). There was no significant relationship between insurance status and cardiovascular mortality.

Conclusions: In a nationally representative sample of individuals with albuminuria, uninsurance and public insurance were associated with increased mortality compared to the private insurance even after controlling for sociodemographic, health status, and health care variables. Improving access to care and the quality of care received may potentially reduce mortality in individuals with evidence of early CKD.
\end{abstract}

Keywords: Albuminuria, Insurance, NHANES, Chronic kidney disease

\section{Background}

It is estimated that about $13 \%$ of US working-age adults are currently uninsured [1]. The Affordable Care Act has the potential to continue to reduce the number of uninsured individuals in the US [2]. Health insurance is an important first step to health care access for these individuals. Uninsured adults receive less appropriate care

\footnotetext{
*Correspondence: msaunder@medicine.bsd.uchicago.edu

${ }^{1}$ University of Chicago Medicine, 5841 S. Maryland, MC 5000, Chicago, IL 60637, USA

Full list of author information is available at the end of the article
}

and have worse outcomes compared to those with insurance [3-5]. More than 10 million adults in the US have albuminuria with preserved estimated glomerular filtration rate. Albuminuria is a well-established risk factor for chronic kidney disease (CKD) progression, cardiovascular events, and death [6-12]. Although lack of health insurance is known to be an important predictor of adverse outcomes among individuals with various medical conditions [3-5], its impact on mortality in adults with albuminuria has not been thoroughly evaluated. Prior work has shown that individuals who belong to racial 
and ethnic minority groups and those of lower socioeconomic status (SES) have a greater risk of albuminuria, and have greater health consequence associated with albuminuria [12-14]. The objective of this study is to evaluate the association of insurance status with all-cause and cardiovascular mortality among working-age adults with albuminuria in the Third National Health and Nutrition Examination Survey, 1988-1994 (NHANES III).

\section{Methods}

\section{Study population}

NHANES III, conducted by the National Center for Health Statistics (NCHS) between 1988 and 1994, is a nationally representative study based on a stratified, clustered, multistage probability sample survey of the civilian, noninstitutionalized population in the US. All participants provided informed consent. Participants underwent a home interview followed by an extensive physical examination and blood and urine sampling at a mobile examination center. Blood and urine samples and blood pressure were obtained during the physical examination. Self-reported information on sociodemographic characteristics and presence of medical conditions was collected during the home interview [15]. The survey protocol was approved by the NCHS institutional review board. Participants 65 years of age and older were excluded due to their low rates of uninsurance due to Medicare eligibility. Our sample included all NHANES III respondents aged 18-64 years with albuminuria who provided information on insurance.

Of the 33,356 NHANES III participants examined, we excluded 19,313 individuals who were pregnant, older than 65 or younger than 18 years. Of the 14,043, men and non-pregnant women between 18 and 65 years, we excluded 2804 individuals due to missing information on UACR, serum creatinine, or insurance status. We excluded 10,298 individuals without albuminuria and seven with eGFR $<15 \mathrm{ml} / \mathrm{min} / 1.73 \mathrm{~m}^{2}$. Our final sample included 934 individuals with UACR $\geq 30 \mathrm{mg} / \mathrm{g}$ (Fig. 1). Compared to individuals in the final cohort, individuals who were excluded due to missing UACR were more likely to be older (50.3 vs 41.9 years, $p<.001$ ), and have an income $<200 \%$ federal poverty level (FPL) (51.2 vs $41.54 \%, p=0.03)$. Compared to individuals in the final cohort, individuals who were excluded because of missing insurance were more likely to be younger ( 35.2 vs 41.9 years, $p<.001)$, be male (49.7 vs $37.4 \%, p<.001$ ), have $<$ high school education (83.5 vs $73.8 \%, p=0.003$ ), and to have an income $<200 \%$ FPL (73.3 vs $41.5 \%$, $p<.001)$ (Data not shown).

\section{Definition of variables}

Albuminuria was defined as a urine albumin-tocreatinine ratio $[\mathrm{UACR}] \geq 30 \mathrm{mg} / \mathrm{g}$. Estimated glomerular

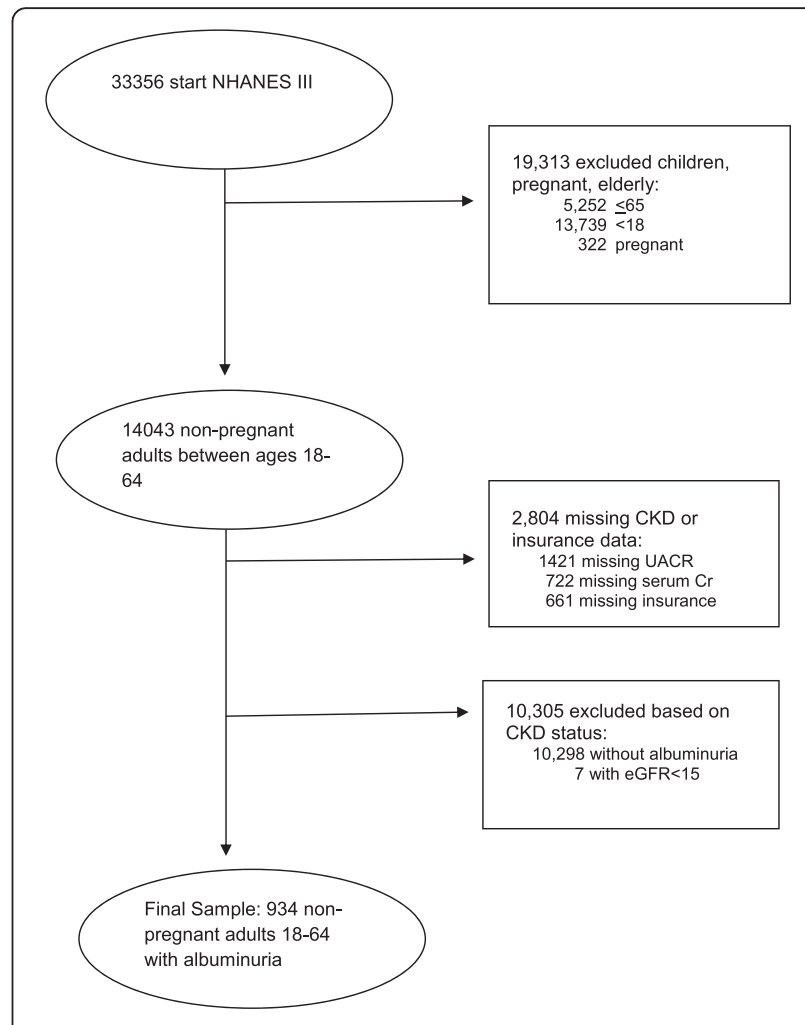

Fig. 1 Study population and exclusions

filtration rate (eGFR) was calculated using the 2012 CKD Epidemiology Collaboration creatinine equation [16]. We used the formula for correction of serum creatinine recommended in the NHANES III Data File Documentation [17]. Hypertension was defined as a $\mathrm{BP}>140 \mathrm{mmHg} />90 \mathrm{mmHg}$ or the use of antihypertensive medications. Diabetes was defined as a history of diabetes, use of insulin or other medication to treat diabetes, a fasting blood glucose level $\geq 126 \mathrm{mg} / \mathrm{dl}$, or a random blood glucose level $\geq 200 \mathrm{mg} / \mathrm{dl}$. Hypercholesterolemia was a total cholesterol concentration $\geq 200 \mathrm{mg} / \mathrm{dL}$. History of cardiovascular disease was defined by an affirmative answer to at least one of the following: history of heart attack, congestive heart failure, or stroke. Insurance status was obtained by patient self-report. At the time of interview patients were asked if they were covered by Medicare, Medicaid, CHAMPUS/VA/military insurance or private insurance in the last month. The answers were categorized as uninsured (none of the above), public insurance (Medicare or Medicaid), or private (all other) insurance.

\section{Outcome ascertainment Mortality follow-up}

Vital status was established using the NHANES III Linked Mortality File which provided follow-up for mortality through December 31, 2006. Probabilistic matching was 
used to link NHANES III participants with the National Death Index (NDI) to ascertain vital status. Matching was based on 12 identifiers for each participant (e.g., Social Security number, sex, and date of birth). Participants who were not matched with any death records were considered to be alive through the follow-up period. Cause of death was assigned by the NCHS based on the International Classification of Diseases, 10th Revision [18]. For this study, cardiovascular mortality was defined as death due to diseases of the heart, essential hypertension and hypertensive kidney disease, cerebrovascular disease, atherosclerosis, and other diseases/disorders of the circulatory system (codes 100-199) [19].

\section{Statistical analysis}

NCHS recommendations were followed to account for stratification and clustering in the survey design, as well as oversampling of ethnic minorities and elderly persons [17]. Continuous variables were expressed as weighted means (standard error) and categorical variables as weighted percentage. Baseline characteristics were compared across insurance status using analysis of variance for continuous variables and chi square for categorical variables.

Weighted age-adjusted all-cause and cardiovascular mortality rates were calculated by 1000 person/year. Cox proportional hazards models were used to determine the association of insurance status with all-cause and cardiovascular mortality in patients with albuminuria adjusting for important covariates in a hierarchical fashion: 1) Model 1: sociodemographic factors (age, gender, race/ethnicity, income, education, and marital status); 2) Model 2: Variables included in Model 1 plus co-morbidities (diabetes mellitus status, cardiovascular disease, smoking, body mass index, and cancer); 3) Model 3: Variables included in Model 2 plus co-morbidity severity (eGFR, systolic blood pressure, serum cholesterol, and hemoglobin A1C); and 4) Model 4: Variables included in Model 3 plus co-morbidity management (use of statin, use of ACEi). In addition, we evaluated for the presence of interaction between insurance status and age, gender, and race/ethnicity on all-cause mortality by adding interaction terms in the fully adjusted models. The proportional hazards assumption of the Cox models was examined using Schoenfeld residuals.

\section{Results}

In our study population (Table 1), the mean age at the time of the interview was 42 years, $63 \%$ of participants were women, $67 \%$ were non-Hispanic White, $16 \%$ were non-Hispanic Black, $6 \%$ were Mexican American and $11 \%$ were of other racial/ethnic background. In addition, approximately $37 \%$ had income less than $200 \%$ FPL, $74 \%$ had less than high school education and $64 \%$ were married. In this sample of NHANES participants, $40 \%$ of participants reported excellent or very good health, $36 \%$ had hypertension, $24 \%$ had diabetes, $44 \%$ had hypercholesterolemia, and $6 \%$ reported cardiovascular disease. The mean eGFR was $102 \mathrm{~mL} / \mathrm{min} /$ $1.73 \mathrm{~m}^{2}$ and median UACR was $71 \mathrm{mg} / \mathrm{g}$. Overall, $4.7 \%$ of the participants had eGFR $<60 \mathrm{ml} / \mathrm{min} / 1.73 \mathrm{~m}^{2}$ and $11.5 \%$ had UACR $\geq 300 \mathrm{mg} / \mathrm{g}$.

The weighted prevalence of uninsured, public insurance and private insurance was 15,18 and $67 \%$, respectively. Compared to individuals with private insurance, those with public insurance or no insurance were significantly more likely to belong to a racial/ethnic minority group, to have income $<200 \% \mathrm{FPL}$, and to have less than high school education $(p<0.05$ for each comparison). Uninsured individuals were younger and less likely to report a usual source of care than their insured counterparts (private or public), all $p<0.05$. Individuals with public insurance differed from their privately (and noninsured counterparts) in that they were more likely to be non-Hispanic Black, to report fair/poor health, to be previously married, and to have hypertension, diabetes or cardiovascular disease, all $p<0.05$. There were no significant differences in mean eGFR, UACR, statin use, or ACE-i use by insurance status.

\section{All-cause and cardiovascular mortality by insurance status and access to care for individuals with albuminuria}

The age-adjusted all-cause mortality rate for individuals with albuminuria was $13.5 / 1000$ person-year (Table 2). Crude rates of all-cause mortality were higher in in the uninsured and individuals with public insurance than in those with private insurance (17.8 and 24.1 vs 10.4 , respectively). A similar pattern was observed with cardiovascular mortality rates.

In fully adjusted models, compared to individuals with private insurance, being uninsured was associated with increased risk for all-cause mortality (HR 2.97, $95 \% \mathrm{CI}$ 1.29-6.85). Compared to individuals with private insurance, having public insurance was also associated with increased mortality (HR 3.65, 95 \% CI 1.74-7.67) (Table 3). There was a suggestion of effect modification by eGFR; however, due to low proportion of individuals with eGFR $<60$ stratified analyses could not be done. We found no interaction between insurance status and race/ethnicity, gender, or age.

The age-adjusted cardiovascular mortality rate for individuals with albuminuria was 5.16/1000 person-year (Table 2). In fully adjusted models, the cardiovascular mortality risk was similar between individuals with private insurance vs uninsured vs public insurance. We found no significant interaction between insurance status and age, gender, race, or eGFR on cardiovascular mortality. 
Table 1 Characteristics of individuals with albuminuria (age <65) overall and stratified by insurance status

\begin{tabular}{|c|c|c|c|c|c|}
\hline Covariates & Overall & Uninsured & Public insurance & Private insurance & $p$ \\
\hline $\mathrm{N}=$ & 903 & 197 & 203 & 503 & \\
\hline Age & $41.90(0.69)$ & $38.26(1.77)$ & $44.88(1.93)$ & $41.90(0.81)$ & 0.04 \\
\hline \multicolumn{6}{|l|}{ Age group } \\
\hline$<45$ & 57.39 & 67.65 & 44.82 & 58.56 & 0.045 \\
\hline $45-65$ & 43.54 & 35.87 & 55.54 & 42.05 & 0.079 \\
\hline \multicolumn{6}{|l|}{ Gender } \\
\hline Male & 37.38 & 31.02 & 36.53 & 39.00 & 0.541 \\
\hline Female & 62.62 & 68.98 & 63.47 & 61.00 & \\
\hline \multicolumn{6}{|l|}{ Race } \\
\hline Non-Hispanic Whites & 66.67 & 51.03 & 49.86 & 74.55 & $<.001$ \\
\hline Non-Hispanic Black & 16.29 & 15.37 & 29.56 & 12.97 & $<.001$ \\
\hline Mexican American & 5.90 & 16.36 & 5.09 & 3.82 & $<.001$ \\
\hline Other & 11.14 & 17.24 & 15.49 & 8.65 & 0.108 \\
\hline \multicolumn{6}{|l|}{ Income } \\
\hline$<100 \mathrm{FPL}$ & 17.54 & 41.76 & 40.55 & 7.27 & $<.0001$ \\
\hline $101-200 \mathrm{FPL}$ & 19.92 & 28.27 & 30.27 & 15.85 & 0.0014 \\
\hline$>200 \mathrm{FBL}$ & 62.54 & 29.97 & 29.18 & 76.88 & $<.001$ \\
\hline FPL (Median, IQR) & $193.8(104.2,331.3)$ & $96.6(58.1,156.4)$ & $95.6(63.25,165.15)$ & $250.6(154.8,379.9)$ & \\
\hline \multicolumn{6}{|l|}{ Education } \\
\hline$<$ High School & 73.79 & 90.17 & 87.93 & 66.45 & $<.001$ \\
\hline$>=$ High School & 26.21 & 9.83 & 12.07 & 33.55 & \\
\hline \multicolumn{6}{|l|}{ Marital Status } \\
\hline Married & 64.10 & 58.98 & 42.90 & 70.84 & $<.001$ \\
\hline Previously Married & 16.70 & 18.04 & 32.50 & 12.23 & $<.001$ \\
\hline Never Married & 19.19 & 22.98 & 24.61 & 16.93 & 0.200 \\
\hline \multicolumn{6}{|l|}{ Self-rated Health } \\
\hline Excellent/Very Good & 40.45 & 28.74 & 25.65 & 46.93 & 0.004 \\
\hline Good & 37.42 & 36.83 & 33.16 & 38.68 & 0.595 \\
\hline Fair/Poor & 22.13 & 34.43 & 41.20 & 14.39 & $<.001$ \\
\hline Usual Source of Care (Yes) & 83.02 & 65.93 & 88.39 & 85.35 & $<.001$ \\
\hline Usual Care Provider (Yes) & 72.95 & 50.58 & 70.03 & 78.64 & $<.001$ \\
\hline Smoking (Yes) & 33.75 & 29.77 & 45.98 & 31.39 & 0.032 \\
\hline Hypertension (Yes) & 36.14 & 34.12 & 45.22 & 34.18 & 0.248 \\
\hline Systolic BP, mm Hg & $127.31(0.96)$ & $127.43(3.08)$ & $128.08(3.03)$ & $127.09(1.28)$ & 0.921 \\
\hline Diastolic BP, mm Hg & $77.80(0.55)$ & $78.34(1.44)$ & $78.19(1.34)$ & $77.57(0.74)$ & 0.670 \\
\hline \multicolumn{6}{|l|}{ BMI } \\
\hline$<30 \mathrm{~kg} / \mathrm{m} 2$ & 65.28 & 62.77 & 62.57 & 66.55 & 0.801 \\
\hline$>=30 \mathrm{~kg} / \mathrm{m} 2$ & 34.72 & 37.23 & 37.43 & 33.45 & \\
\hline Diabetes (Yes) & 23.60 & 25.44 & 36.05 & 19.91 & 0.010 \\
\hline $\mathrm{HbA1C}$ & $6.02(0.10)$ & $6.16(0.18)$ & $6.21(0.18)$ & $5.94(0.14)$ & 0.240 \\
\hline History of CVD (Yes) & 5.53 & 5.23 & 12.39 & 3.79 & 0.010 \\
\hline ACE Use (Yes) & 13.19 & 20.88 & 18.72 & 10.62 & 0.097 \\
\hline Cholesterol (Yes) & 43.80 & 49.71 & 40.23 & 43.45 & 0.427 \\
\hline Statin Use (Yes) & 4.48 & 10.33 & 7.52 & 2.81 & 0.277 \\
\hline
\end{tabular}


Table 1 Characteristics of individuals with albuminuria (age <65) overall and stratified by insurance status (Continued)

\begin{tabular}{|c|c|c|c|c|c|}
\hline Mean eGFR & $101.61(1.18)$ & $105.40(3.20)$ & $97.87(4.02)$ & $101.76(1.48)$ & 0.250 \\
\hline eGFR < $60(\%)$ & 4.74 & 3.46 & 9.30 & 3.81 & 0.177 \\
\hline Albuminuria Median (IQR) & $70.92(43.02,154.42)$ & $69.48(40.47,123.40)$ & $98.28(46.51,265.43)$ & $67.09(42.46,143.50)$ & \\
\hline \multicolumn{6}{|l|}{ UACR } \\
\hline $30-299$ & 88.52 & 90.99 & 83.47 & 89.32 & 0.288 \\
\hline$>=300$ & 11.48 & 9.01 & 16.53 & 10.68 & \\
\hline
\end{tabular}

Note: History of CVD is defined as has history of heart failure or stroke

\section{Discussion}

In this nationally representative sample of individuals with albuminuria and preserved eGFR, we found that both uninsurance and public insurance were associated with a significantly higher risk for mortality compared to private insurance. Uninsured individuals with albuminuria had nearly a three-fold higher risk for all-cause mortality than their privately insured counterparts after controlling for socioeconomic and clinical characteristic. Similar to our findings, the Kidney Early Evaluation Program (KEEP), a community-based health screening program, found an increased risk for death in uninsured participants with CKD stages 1-2 as compared to those with private insurance [20]. Our study provides corroborative evidence in a sample representative of the US population.

We found that in a working age, US population with albuminuria, $15 \%$ were uninsured, $18 \%$ had public insurance, and $67 \%$ had private insurance. Uninsurance was higher than a previous examination of uninsured adults with CKD because we looked only at individuals younger than 65 whom comprise over $90 \%$ of the uninsured with CKD [21]. Our uninsurance rate was closer to Wilper et al. who also used NHANES to examine uninsurance in working age adults $[5,22]$.

In the general population, the association of uninsurance with increased risk for mortality is well established $[5,23,24]$. However, the relationship between insurance status and outcomes has not been as well studied in the CKD population. In a cross-sectional analysis of data from NHANES, uninsured individuals with CKD were less likely to have controlled hypertension or to receive

Table 2 Mortality rate by insurance status for individuals with albuminuria

\begin{tabular}{lcc}
\hline Variable $(N=915)$ & $\begin{array}{l}\text { Age-adjusted mortality } \\
\text { rate (All-cause) per 1,000 } \\
\text { person-year }\end{array}$ & $\begin{array}{l}\text { Age-adjusted mortality } \\
\text { rate }(\mathrm{CV}) \text { per 1,000 } \\
\text { person-year }\end{array}$ \\
\hline Overall & 13.53 & 5.16 \\
$\begin{array}{l}\text { Insurance status } \\
\text { (ref: Private) }\end{array}$ & 10.39 & 4.14 \\
Uninsured & 17.76 & 7.02 \\
Public & 24.06 & 8.06 \\
\hline
\end{tabular}

an angiotensin-converting enzyme inhibitor (ACEi) than those with insurance $[21,25]$. At the time of our study, rates of both ACEi and statin use were low in the general population [26, 27]. Although we did not find a significant difference in rates of ACEi use across insurance categories, adding ACEi and statin to our final model increased both the magnitude and significance of the association between uninsurance and mortality. These findings suggest that $\mathrm{ACE} /$ statin are important mediators of the association or that they are potentially in the causal pathway.

Publicly insured individuals with albuminuria also had a significantly higher all-cause mortality rate than their privately insured counterparts. This finding was true both in the crude model and after adjusting for socioeconomic and clinical characteristics. For most workingage adults, eligibility for public insurance is dependent on income and poor health [28]. In a national survey data study, Sorlie et al. also reported increased mortality in individuals with public insurance as compared to those with employer provided insurance [29]. Sorlie also found that in certain groups, public insurance was associated with increased mortality compared to uninsurance [29].

Our findings that health insurance is an important predictor of mortality in individuals with kidney disease, many of whom had early signs of kidney disease, has important implications in the current climate of healthcare reform. The number of uninsured in the US has decreased due both to the economic recovery and implementation of Affordable Care Act. The uninsurance rate for working age adults, 18-64 years old, was estimated to be $12.4 \%$ in late 2014 [1]. Due to targeted policies, poor working adults saw the largest gains in insurance coverage, particularly in Medicaid expansion states [1]. However, the uninsured have gained coverage through a combination of expansion of employer-based coverage, health insurance exchanges, extended parental coverage, and Medicaid expansion [30]. More individuals may potentially benefit given the current eligibility, and requirement, for nearly all working age adults to obtain insurance. In addition, the association of public insurance with increased mortality will likely be reduced as eligibility is based on income rather than health status. 
Table 3 Risk for all-cause mortality by insurance status and access to care for individuals with albuminuria

\begin{tabular}{|c|c|c|c|c|}
\hline & All-cause mortality & $P$ & CV mortality & $P$ \\
\hline $\mathrm{N}=$ & 913 & & 913 & \\
\hline \multicolumn{5}{|l|}{ Unadjusted HR } \\
\hline No Insurance V Private (REF) & $1.30(0.77,2.21)$ & 0.316 & $1.30(0.50,3.40)$ & 0.581 \\
\hline Public V Private & $2.75(1.61,4.68)$ & $<.001$ & $2.30(1.12,4.72)$ & 0.024 \\
\hline \multicolumn{5}{|l|}{ HR, Model $1^{\mathrm{a}}$} \\
\hline No Insurance V Private (REF) & $1.80(0.74,4.34)$ & 0.189 & $1.96(0.66,5.77)$ & 0.219 \\
\hline Public V Private & $1.70(0.87,3.34)$ & 0.118 & $1.73(0.63,4.76)$ & 0.285 \\
\hline \multicolumn{5}{|l|}{ HR, Model $2^{b}$} \\
\hline No Insurance V Private (REF) & $1.86(0.71,4.87)$ & 0.199 & $1.88(0.46,7.58)$ & 0.370 \\
\hline Public V Private & $1.66(0.81,3.43)$ & 0.165 & $1.48(0.47,4.65)$ & 0.492 \\
\hline \multicolumn{5}{|l|}{ HR, Model $3^{c}$} \\
\hline No Insurance V Private (REF) & $1.90(0.83,4.34)$ & 0.125 & $1.95(0.56,6.85)$ & 0.288 \\
\hline Public V Private & $1.87(1.01,3.46)$ & 0.046 & $1.57(0.52,4.73)$ & 0.417 \\
\hline \multicolumn{5}{|l|}{ HR, Model $4^{\mathrm{d}}$} \\
\hline No Insurance V Private (REF) & $2.97(1.29,6.85)$ & 0.012 & $2.15(0.44,10.46)$ & 0.337 \\
\hline Public V Private & $3.65(1.74,7.67)$ & $<.001$ & $3.21(0.77,13.43)$ & 0.107 \\
\hline
\end{tabular}

${ }^{a}$ Model 1: Sociodemographic: age, gender, race/ethnicity, PIR (poverty income ratio), education, marital status

${ }^{\mathrm{b}}$ Model 2: Model 1+ Diabetes $(\mathrm{Y} / \mathrm{N})$, Cardiovascular disease $(\mathrm{Y} / \mathrm{N})$, smoking $(\mathrm{Y} / \mathrm{N})$, Body mass index, cancer $(\mathrm{y} / \mathrm{n})$

${ }^{c}$ Model 3: Model 2+ estimated glomerular filtration rate (eGFR), systolic blood pressure, cholesterol $(<200 \mathrm{mg} / \mathrm{dL},>200 \mathrm{mg} / \mathrm{dL} \mathrm{HgbA1c}(<7,>7)$

${ }^{\mathrm{d}}$ Model 4: Model $3+$ statin(Y/N), angiotension converting enzyme (ACE)-Inhibitor (yes/no)

We found a less convincing relationship between insurance status and cardiovascular mortality. The uninsured did not have a significantly different CV mortality than their privately insured counterparts. This finding seems surprising given the importance of medical care in managing cardiovascular risk factors and intervening on cardiovascular events. One possible explanation is that uninsured individuals with CKD and CV disease may remain uninsured for less time than their healthier counterparts. Due either to the number or severity of $\mathrm{CV}$ risk factors, they are more likely to meet criteria for public insurance. One final explanation is that since everyone regardless of insurance status is eligible for medical care during a cardiovascular emergency, the effect of insurance status on access to life-saving CV care is less important.

Our study had several limitations. Because of the design of NHANES, we only had access to a single UACR and eGFR determination, rather than the multiple measures. However, prior studies have used this method to ascertain CKD $[21,26]$. In addition, we were able to determine insurance status only at a single point in time. Patients may have had only a limited period of uninsurance or insured patients may have subsequently lost their insurance. Nonetheless, our classification of insurance status based on a single assessment was associated with significant differences in long-term outcomes.

\section{Conclusions}

In conclusion, in a nationally representative sample of individuals with albuminuria and preserved eGFR, both uninsurance and public insurance were associated with increased mortality compared to private insurance, even after controlling for sociodemographic, health status, and health care variables. Given the burdens of kidney disease both to the affected individuals, families, and the health care system, we must wait to see if efforts to provide comprehensive health care-by improving access to care and the quality of care received-will be able to help to reduce costs, morbidity and mortality for those with early evidence of CKD.

\section{Availability of data and materials}

The datasets supporting the conclusions of this article are available in the National Center for Health Statistics data repository. Third National Health and Nutrition Examination Survey (NHANES III) data is available at doi.org/ 10.3886/ICPSR02231.v1 and http://www.cdc.gov/nchs/ nhanes/nhanes3/data_files.htm\# with linkage to the National Death Index at http://www.cdc.gov/nchs/data access/data_linkage/mortality.htm.

\section{Abbreviations}

ACEi: angiotension converting enzyme inhibitor; BP: blood pressure; $\mathrm{Cl}$ : confidence interval; CKD: chronic kidney disease; CV: cardiovascular; eGFR: estimated filtration rate; FPL: federal poverty level; NCHS: National Center for Health Statistics; NHANES: National Health and Nutrition 
Examination Study; UACR: urine albumin-to-creatinine ratio; US: United States.

\section{Competing interests}

Financial and Other Disclosure: Drs. Saunders, Ricardo, Chen, Chin and Lash have no conflicts of interest to disclose.

\section{Authors' contributions}

MS participated in the conception and design of the study, interpretation of the data and drafting of the work; AC participated in design of the work and revising it critically for important intellectual content; JC participated in the acquisition and analysis of the data; MC participated in the interpretation of the data and revising it critically for important intellectual content; JL participated in the conception and design of the work, interpretation of the results, and revising it for critically important intellectual content. All authors read and approved the final manuscript.

\section{Acknowledgements}

Support: The authors acknowledge support from a Pilot and Feasibility Award P30 DK092949 (Saunders), K23 DK103111 (Saunders), K24 DK092290 (Lash), K23 DK094829 (Ricardo), P30 DK092949 (Chin), K24 DK071933 (Chin).

\section{Author details}

${ }^{1}$ University of Chicago Medicine, 5841 S. Maryland, MC 5000, Chicago, IL 60637, USA. 'University of Illinois at Chicago, 820 Wood St, MC 793, Chicago, IL 60612, USA. ${ }^{3}$ University of Chicago Medicine, 5841 S. Maryland, MC 2007, Chicago, IL 60637, USA.

\section{Received: 22 June 2015 Accepted: 1 March 2016}

Published online: 09 March 2016

\section{References}

1. Long SK, Karpman M, Shartzer A, Wissoker D, Kenney GM, Zuckerman S, Anderson N, Hempstead K. Taking stock: Health insurance coverage under the ACA as of September 2014. 2014.

2. Schoen C, Doty MM, Robertson $\mathrm{RH}$, Collins SR. Affordable care act reforms could reduce the number of underinsured US adults by 70 percent. Health Aff. 2011;30(9):1762-71.

3. McWilliams JM, Zaslavsky AM, Meara E, Ayanian JZ. Health insurance coverage and mortality among the near-elderly. Health Aff. 2004;23(4):223-33.

4. Wilper AP, Woolhandler S, Lasser KE, McCormick D, Bor DH, Himmelstein DU. Hypertension, diabetes, and elevated cholesterol among insured and uninsured U.S. adults. Health Aff. 2009:28(6):w1151-9.

5. Wilper AP, Woolhandler S, Lasser KE, McCormick D, Bor DH, Himmelstein DU. Health insurance and mortality in US adults. Am J Public Health. 2009;99(12):2289-95.

6. Hemmelgarn BR, Manns BJ, Lloyd A, James MT, Klarenbach S, Quinn RR Wiebe N, Tonelli M. Alberta kidney disease network: Relation between kidney function, proteinuria, and adverse outcomes. JAMA. 2010;303(5):423-9.

7. Hallan SI, Ritz E, Lydersen S, Romundstad S, Kvenild K, Orth SR. Combining GFR and albuminuria to classify CKD improves prediction of ESRD. J Am Soc Nephrol. 2009;20(5):1069-77.

8. Peralta CA, Shlipak MG, Judd S, Cushman M, McClellan W, Zakai NA, Safford MM, Zhang X, Muntner P, Warnock D. Detection of chronic kidney disease with creatinine, cystatin $C$, and urine albumin-to-creatinine ratio and association with progression to end-stage renal disease and mortality. JAMA. 2011;305(15):1545-52.

9. Levey AS, Coresh J. Chronic kidney disease. Lancet. 2012:379(9811):165-80.

10. Ninomiya T, Perkovic V, de Galan BE, Zoungas S, Pillai A, Jardine M, Patel A, Cass A, Neal B, Poulter N, Mogensen CE, Cooper M, Marre M, Williams B, Hamet P, Mancia G, Woodward M, Macmahon S, Chalmers J. Albuminuria and kidney function independently predict cardiovascular and renal outcomes in diabetes. J Am Soc Nephrol. 2009;20(8):1813-21.

11. Klausen $K$, Borch-Johnsen $K$, Feldt-Rasmussen B, Jensen $G$, Clausen P, Scharling $H$, Appleyard M, Jensen JS. Very low levels of microalbuminuria are associated with increased risk of coronary heart disease and death independently of renal function, hypertension, and diabetes. Circulation. 2004; 110(1):32-5

12. Arnlöv J, Evans JC, Meigs JB, Wang TJ, Fox CS, Levy D, Benjamin EJ, D'Agostino RB, Vasan RS. Low-grade albuminuria and incidence of cardiovascular disease events in nonhypertensive and nondiabetic individuals the framingham heart study. Circulation. 2005;112(7):969-75.

13. Jolly SE, Burrows NR, Chen SC, Li S, Jurkovitz CT, Narva AS, Norris KC, Shlipak MG. Racial and ethnic differences in albuminuria in individuals with estimated GFR greater than $60 \mathrm{~mL} / \mathrm{min} / 1.73 \mathrm{~m}(2)$ : results from the Kidney Early Evaluation Program (KEEP). Am J Kidney Dis. 2010;55(3):S15-22.

14. McClellan WM, Warnock DG, Judd S, Muntner P, Kewalramani R, Cushman M, McClure LA, Newsome BB, Howard G. Albuminuria and racial disparities in the risk for ESRD. J Am Soc Nephrol. 2011;22(9):1721-8.

15. Plan and operation of the Third National Health and Nutrition Examination Survey, 1988-94. Series 1: programs and collection procedures. Vital Health Stat 1. 1994;(32):1-407.

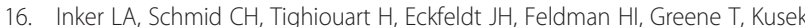
JW, Manzi J, Van Lente F, Zhang YL, Coresh J, Levey AS. Estimating glomerular filtration rate from serum creatinine and cystatin C. N Engl J Med. 2012;367(1):20-9.

17. NHANES III Laboratory Date File Documentation. Ages one and older. Hyattsville, MD: National Center for Health Statistics; 1996.

18. The Third National Health and Nutrition Examination Survey (NHANES III) Linked Mortality File, Mortality follow-up through 2006: Matching Methodology. National Center for Health Statistics, Office of Analysis and Epidemiology. http://www.cdc.gov/nchs/data/datalinkage/matching_ methodology_nhanes3_final.pdf. Accessed March 6, 2013.

19. Anderson RN, Minino AM, Hoyert DL, Rosenberg HM. Comparability of cause of death between ICD-9 and ICD-10: preliminary estimates. Natl Vital Stat Rep. 2001:49:1-32

20. Jurkovitz CT, Li S, Norris KC, Saab G, Bomback AS, Whaley-Connell AT, McCullough PA. Association between lack of health insurance and risk of death and ESRD: results from the Kidney Early Evaluation Program (KEEP). Am J Kidney Dis. 2013;61(4 Suppl 2):S24-32.

21. Hall YN, Rodriguez RA, Boyko EJ, Chertow GM, O'Hare AM. Characteristics of uninsured Americans with chronic kidney disease. J Gen Intern Med. 2009; 24(8):917-22.

22. Wilper AP, Woolhandler S, Lasser KE, McCormick D, Bor DH, Himmelstein DU. A national study of chronic disease prevalence and access to care in uninsured U.S. adults. Ann Intern Med. 2008;149(3):170-6.

23. Franks P, Clancy CM, Gold MR. Health insurance and mortality. Evidence from a national cohort. JAMA. 1993;270(6):737-41.

24. Franks P, Gold MR, Clancy CM. Use of care and subsequent mortality: the importance of gender. Health Serv Res. 1996;31(3):347-63.

25. Evans K, Coresh J, Bash LD, Gary-Webb T, Köttgen A, Carson K, Boulware LE. Race differences in access to health care and disparities in incident chronic kidney disease in the US. Nephrol Dial Transplant. 2011;26(3):899-908.

26. Plantinga LC, Miller ER, Stevens LA, Saran R, Messer K, Flowers N, Geiss L, Powe NR. Blood pressure control among persons without and with chronic kidney disease: US trends and risk factors 1999-2006. Hypertension. 2009;54(1):47-56.

27. Mann D, Reynolds K, Smith D, Muntner P. Trends in statin use and lowdensity lipoprotein cholesterol levels among US adults: Impact of the 2001 National cholesterol education program guidelines. Ann Pharmacother. 2008;42(9):1208-15.

28. Hadley J. Sicker and poorer — The consequences of being uninsured: A review of the research on the relationship between health insurance, medical care use, health, work, and income. Med Care Res Rev. 2003;60(2 suppl):3S-75

29. Sorlie PD, Johnson NJ, Backlund E, Bradham DD. Mortality in the uninsured compared with that in persons with public and private health insurance. Arch Intern Med. 1994;154(21):2409-16.

30. Carman KG, Eibner C. Changes in health insurance enrollment since 2013 : Evidence from the RAND health reform opinion study. Santa Monica, CA: RAND Corporation; 2014. http://www.rand.org/pubs/research_reports/RR656. 